\title{
PREVALÊNCIA DE ALTERAÇÃo MORFOLÓGICA dO PROCESSO ESTILÓIDE EM PACIENTES COM DESORDEM TEMPOROMANDIBULAR*
}

\author{
Simone Maria Ragone Guimarães ${ }^{1}$, Antonio Carlos Pires Carvalho ${ }^{2}$, Josemar Parreira Guimarães ${ }^{3}$, \\ Marden Batista Gomes ${ }^{4}$, Mariana de Mello Melquíades Cardoso ${ }^{4}$, Henrique Nogueira Reis ${ }^{4}$
}

Resumo OBJETIVO: Avaliar, radiograficamente, a prevalência de alterações morfológicas do processo estilóide em pacientes com desordens temporomandibulares. MATERIAIS E MÉTODOS: Foram analisadas 1.500 radiografias panorâmicas da articulação temporomandibular de pacientes de ambos os sexos e sem limitação de idade, que foram atendidos pelo Serviço de Desordem Temporomandibular da Faculdade de Odontologia da Universidade Federal de Juiz de Fora, MG, no período de 1997 a 2003. RESULTADOS: Oitenta e três $(5,53 \%)$ dos pacientes da amostra apresentaram pelo menos um dos lados da articulação com alteração morfológica do processo estilóide, sendo 74 do sexo feminino e 9 do sexo masculino, concentrados na faixa dos 41 a 50 anos de idade $(32,5 \%)$. Com relação ao tipo morfológico do processo estilóide, verificaram-se 113 alongados, 21 pseudo-articulados e 19 segmentados. Constatou-se, também, que as alterações morfológicas do processo estilóide desenvolvem-se de forma simétrica. CONCLUSÃO: Em pacientes com desordem temporomandibular as alterações do processo estilóide ocorrem de forma diferenciada e de maneira simétrica em cada paciente, independentemente do sexo e da idade.

Unitermos: Processo estilóide; Desordem temporomandibular; Radiografia.

Abstract Prevalence of morphological alterations of the styloid process in patients with temporomandibular joint disorder.

OBJECTIVE: To assess radiographically the prevalence of morphological alterations of the styloid process in patients with temporomandibular joint disorders. MATERIALS AND METHODS: We have analyzed 1,500 panoramic x-ray films of temporomandibular joint from both male and female patients with no age limit, who were attended in the Service of Temporomandibular Joint Disorder of Faculty of Odontology at Universidade Federal de Juiz de Fora, MG, Brazil, in the period between 1997 and 2003. RESULTS: Eighty-three (5.53\%) of the analyzed patients ( 74 female and 9 male), predominantly in the age range between 41 and 50 years $(32.5 \%)$, presented with, at least, one of the joint sides with morphological alteration of the styloid process. As far as the type of styloid process in concerned, 113 elongated, 21 pseudoarticulated and 19 segmented were observed. Also, it was noticed that the morphological alterations of the styloid process develop in a symmetrical fashion. CONCLUSION: In individuals with temporomandibular joint disorder, styloid process alterations occur symmetrically and in a different way for each patient, independently from sex and age. Keywords: Styloid process; Temporomandibular joint disorder; Radiography.

\section{INTRODUÇÃO}

Para o diagnóstico e etiologia das disfunções craniomandibulares, é necessário o conhecimento da anatomia e fisiologia das articulações temporomandibulares e estruturas adjacentes, tanto a parte óssea quanto as partes musculares, ligamentares e o disco articular, objetivando interpretar as alterações morfológicas e suas doen-

* Trabalho desenvolvido no Serviço de Diagnóstico e Orientação a Pacientes com Desordens Temporomandibulares da Faculdade de Odontologia da Universidade Federal de Juiz de Fora, Juiz de Fora, MG, como parte dos requisitos para conclusão do Mestrado em Ciências - Área Radiologia - na Faculdade de Medicina da Universidade Federal do Rio de Janeiro.

1. Cirurgiã-dentista, Especialista e Mestre em Radiologia, Professora de Radiologia da Faculdade de Odontologia da Universidade Federal de Juiz de Fora.

2. Médico, Doutor em Radiologia, Professor Adjunto da Faculdade de Medicina da Universidade Federal do Rio de Janeiro. ças $^{(\mathbf{1})}$. Entre as disfunções craniomandibulares, pode-se citar as desordens temporomandibulares e a síndrome de Eagle, também denominada síndrome do processo estilóide ou síndrome da artéria carótida ${ }^{(\mathbf{1})}$.

A síndrome de Eagle compreende as alterações anatômicas do processo estilóide e/ou ligamento estilohióideo, que podem gerar manifestações clínicas que se aproximam das desordens temporomandibula-

3. Cirurgião-dentista, Mestre e Doutor em Ortodontia, Coordenador do Serviço-ATM da Faculdade de Odontologia da Universidade Federal de Juiz de Fora.

4. Cirurgiōes-dentistas, Membros da Equipe Interdisciplinar do Senviço-ATM da Faculdade de Odontologia da Universidade Federal de Juiz de Fora.

Endereço para correspondência: Dra. Simone Maria Ragone Guimarães. Avenida Rio Branco, 2406/1103, Centro. Juiz de Fora, MG, 36016-200. E-mail: odontoimagem@acessa.com Recebido para publicação em 27/9/2005. Aceito, após revisão, em 20/3/2006. res. Por sua vez, as desordens temporomandibulares estão relacionadas com muitas características estruturais e funcionais complexas da articulação temporomandibular e apresentam sintomas semelhantes à síndrome de Eagle ${ }^{(2)}$.

Vários sintomas têm sido atribuídos à síndrome de Eagle, incluindo dor cervical, otalgia, dor e sensação de "corpo estranho" na garganta, dor ao mudar a posição da cabeça, cefaléia, dor na região cervicofacial, dor durante a deglutição, dor nos ombros, entre outros ${ }^{(\mathbf{3})}$. Dentre os sintomas apresentados pelos pacientes com desordem temporomandibular encontram-se mais presentes: artralgia, estalido articular, cefaléia, otalgia, dor muscular, zumbido, dificuldade de abrir a boca, movimentos excursivos limitados, e outros ${ }^{(4)}$. 
O processo estilóide é um osso (esporão) delgado e cilíndrico ${ }^{(5)}$. Está localizado acima da artéria carótida comum, entre as ramificações externa e interna, imediatamente próximo à veia jugular interna e ao nervo facial ${ }^{(\mathbf{1})}$. O comprimento do processo estilóide pode variar, porém apresenta média de $25 \mathrm{~mm}$; pode também estar ausente ${ }^{(\mathbf{6})}$, e também apresentar-se alongado.

Langlais et al. ${ }^{(7)}$ classificaram em três tipos os alongamento do processo estilóide e/ou calcificação do ligamento estilohióideo, de acordo com a aparência radiográfica: alongado - o processo estilóide e o ligamento aparecem como uma estrutura contínua de 2,5 a 3,2 cm de comprimento; pseudo-articulado - o processo parece estar unido ao ligamento estilomandibular ou estilohióideo, por uma única pseudo-articulação, normalmente localizada acima do ângulo da mandíbula; segmentado - o processo estilóide e ligamentos consistem em vários segmentos mineralizados.

O diagnóstico diferencial entre a síndrome de Eagle e a disfunção da articulação temporomandibular pode ser feito pela história clínica, palpação digital do processo estilóide na área da fossa amigdaliana, infiltração de anestesia local ${ }^{(\mathbf{5 , 8})}$, bem como pela visualização do processo estilóide no exame radiográfico ou na tomografia computadorizada $^{(9)}$. Além da palpação, pode-se avaliar a abertura bucal, as inspeções intra e extrabucal, e a observação de pontos ál$\operatorname{gicos}^{(\mathbf{1})}$. Em 1937, Eagle ${ }^{(\mathbf{1 0 )}}$ sugeriu a confirmação diagnóstica através da tríade clássica, composta de sensação de corpo estranho na garganta, massa endurecida palpável na região tonsilar e imagem radiográfica com alongamento do processo estilóide.

Existem diversos métodos de imagens radiográficas para visualização das articulações temporomandibulares, como a panorâmica, a panorâmica modificada, a transfacial, a transcranial, a transorbital, e a tomografia ${ }^{(3)}$. Entretanto, diversos autores $^{(3,5,11,12)}$ recomendam a radiografia panorâmica para visualização e análise do processo estilóide, bem como suas variações de tamanho e forma. Arellano ${ }^{(\mathbf{1 3})}$ afirmou que a radiografia panorâmica apresenta limitações como ampliação, sobreposição de imagens e falta de nitidez.

Assim sendo, relacionando as desordens temporomandibulares e a síndrome de
Eagle, este estudo se propõe a avaliar, radiograficamente, em pacientes com desordem temporomandibular, a presença de alterações morfológicas do processo estilóide e/ou ligamento estilohióideo, passíveis de interferências no diagnóstico desta desordem articular.

\section{MATERIAIS E MÉTODOS}

Foram avaliadas 1.500 radiografias das articulações temporomandibulares de pacientes de ambos os sexos e sem limitação da faixa etária, que foram atendidos no Serviço de Diagnóstico e Orientação de Pacientes com Desordens Temporomandibulares da Faculdade de Odontologia da Universidade Federal de Juiz de Fora, no período de agosto de 1997 a março de 2003. Todas foram realizadas em aparelho modelo Ro- tograph Plus (Dabi Atlante), com miliamperagem fixa $(10 \mathrm{~mA})$ e quilovoltagem variável de acordo com o porte físico do paciente (variando de $60 \mathrm{kVp}$ a $85 \mathrm{kVp}$ ). Utilizou-se filme da marca Kodak ${ }^{\circledR}$, com chassi curvo e placa intensificadora de tamanhos $12,7 \times 30,5 \mathrm{~cm}$. O processamento foi realizado manualmente pelo método tempo/temperatura.

As radiografias foram analisadas e selecionadas as que apresentavam alteração morfológica do processo estilóide em pelo menos um dos lados da articulação. Os tipos (variações) morfológicos foram avaliados e classificados de acordo com os critérios preconizados por Langlais et al. ${ }^{(7)}$ em: normal, alongado, segmentado e pseudo-articulado (Figura 1).

Para avaliar a associação entre as variáveis qualitativas nominais (categóricas) foi

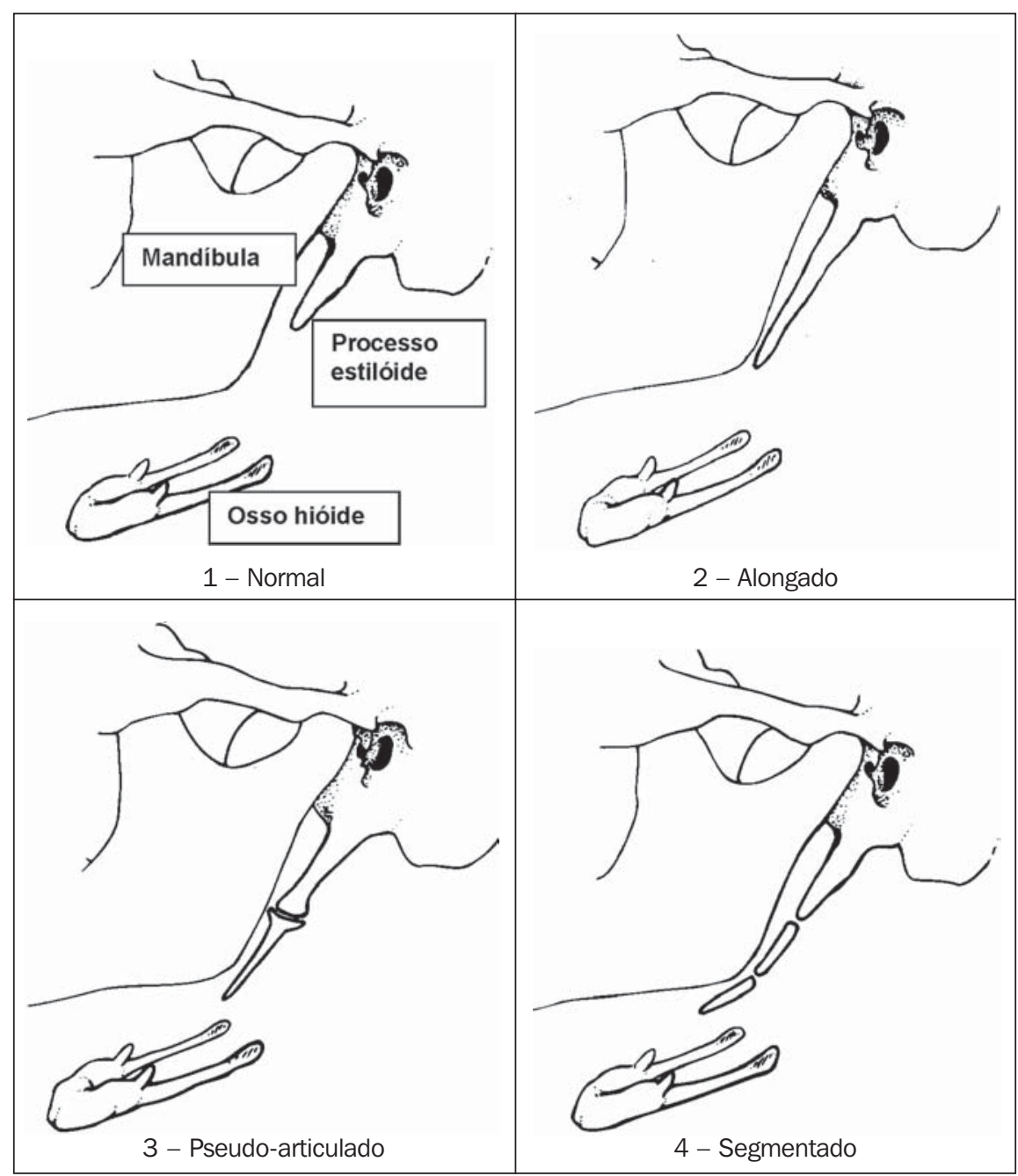

Figura 1. Esquema da classificação das alterações morfológicas do processo estilóide ${ }^{(7)}$ (modificado pela autora). 
utilizado o coeficiente de correlação V de Cramer.

\section{RESULTADOS}

Do grupo de 1.500 radiografias, 83 pacientes $(5,53 \%)$ apresentaram alteração morfológica de pelo menos um dos processos estilóides, sendo mais prevalente no sexo feminino (74 pacientes), ou seja, $89,2 \%$ da amostra, e em somente 9 pacientes do sexo masculino. Apesar dessa distribuição por sexo, não houve, percentualmente, predominância de um sexo em relação ao outro, apesar de a amostra constar de mais mulheres.

A distribuição por faixa etária pode ser vista na Tabela 1, em que se verifica maior concentração de pacientes $(32,5 \%)$ com idade variando entre 41 e 50 anos. Não foi verificado nenhum paciente com idade menor que 10 anos que tivesse alteração morfológica do processo estilóide.

A classificação geral do padrão morfológico do processo estilóide pode ser vista na Tabela 2, sendo o tipo alongado o mais freqüente $(n=113)$, com 59 do lado direito e 54 do lado esquerdo.

Em relação à simetria (Tabela 3) do tipo morfológico do processo estilóide, constatou-se que $56,6 \%(n=47)$ são do tipo alongado bilateral (2/2). Em seguida, os mais incidentes foram o tipo alongado direito e normal esquerdo $(2 / 1)$, com $12 \%(n=10)$ da amostra, e o pseudo-articulado bilateral (3/3), com 9 pacientes $(10,8 \%)$.

\section{DISCUSSÃO}

Muitos estudos ${ }^{(\mathbf{1}, 2,4,14)}$ têm sido realizados para melhor compreensão do funcionamento das desordens associadas às articu-

Tabela 1 Distribuição por faixa etária da amostra de estudo $(n=83)$.

\begin{tabular}{|l|c|c|}
\hline Faixa etária & $\begin{array}{c}\text { Freqüência } \\
(\mathrm{n})\end{array}$ & $\begin{array}{c}\text { Percentual } \\
(\%)\end{array}$ \\
\hline 1 (até 10 anos) & 0 & 0 \\
2 (11 a 20 anos) & 5 & 6 \\
3 (21 a 30 anos) & 12 & 14,5 \\
4 (31 a 40 anos) & 14 & 16,9 \\
5 (41 a 50 anos) & 27 & 32,5 \\
6 (51 a 60 anos) & 13 & 15,7 \\
7 (61 a 82 anos) & 12 & 14,5 \\
\hline \multicolumn{1}{|c|}{$n$} & 83 & 100 \\
\hline
\end{tabular}

Tabela 2 Alteração morfológica do processo estilóide da amostra de estudo $(n=83)$.

\begin{tabular}{|l|r|rr|rr|}
\hline \multirow{2}{*}{$\begin{array}{l}\text { Tipo morfológico do processo } \\
\text { estilóide }\end{array}$} & $\begin{array}{c}\text { Freqüência total } \\
(\mathrm{n})\end{array}$ & \multicolumn{2}{|c|}{ Lado direito } & \multicolumn{2}{|c|}{ Lado esquerdo } \\
\cline { 3 - 6 } & 13 & 3 & 3,6 & 10 & 12,0 \\
\hline 1 - Normal & 113 & 59 & 71,1 & 54 & 65,1 \\
2 - Alongado & 21 & 9 & 10,8 & 12 & 14,5 \\
3 - Pseudo-articulado & 19 & 12 & 14,5 & 7 & 8,4 \\
4 - Segmentado & 166 & 83 & 100,0 & 83 & 100,0 \\
\hline Total & 19 & & &
\end{tabular}

Tabela 3 Classificação bilateral do tipo morfológico do processo estilóide $(n=83)$.

\begin{tabular}{|l|c|c|}
\hline $\begin{array}{l}\text { Tipo morfológico do processo estilóide } \\
\text { direito/esquerdo }\end{array}$ & $\begin{array}{c}\text { Freqüência } \\
(\mathrm{n})\end{array}$ & $\begin{array}{c}\text { Percentual } \\
(\%)\end{array}$ \\
\hline 1/2 - Normal/alongado & 2 & 2,4 \\
1/3 - Normal/pseudo-articulado & 1 & 1,2 \\
2/1 - Alongado/normal & 10 & 12,0 \\
2/2 - Alongado/alongado & 47 & 56,6 \\
2/3 - Alongado/pseudo-articulado & 1 & 1,2 \\
2/4 - Alongado/segmentado & 1 & 1,2 \\
3/3 - Pseudo-articulado bilateral & 9 & 10,8 \\
$4 / 2$ - Segmentado/alongado & 5 & 6,0 \\
$4 / 3$ - Segmentado/pseudo-articulado & 1 & 1,2 \\
$4 / 4$ - Segmentado bilateral & 6 & 7,2 \\
\hline Total & 83 & 100,0 \\
\hline
\end{tabular}

lações temporomandibulares, cujas alterações morfológicas podem traduzir-se em uma gama de sinais e sintomas que dificultam o diagnóstico e, conseqüentemente, as condutas terapêuticas $^{(2)}$.

Apesar de diversos estudos realizarem a mensuração do tamanho do processo estilóide, não foi proposta deste realizá-la, uma vez que a radiografia utilizada nas análises (panorâmica modificada para a articulação temporomandibular) apresenta distorções e ampliações no seu processo de obtenção ${ }^{(13)}$. Entretanto, em diversos estudos publicados ${ }^{(\mathbf{3 , 5}, \mathbf{1 1}, \mathbf{1 2})}$ a radiografia panorâmica foi utilizada para observação e análise do processo, pois este exame é de fácil execução e, de certa forma, acessível aos pacientes. Para Arellano ${ }^{(\mathbf{1 3})}$, Basekim et $a l .{ }^{(6)}$ e Heitz et al. ${ }^{(15)}$, a tomografia computadorizada é o método mais eficaz para avaliar o comprimento e as alterações morfológicas do processo, uma vez que o exame disponibiliza reconstruções bi e tridimensionais de toda a articulação temporomandibular $^{(\mathbf{1 6})}$. Entretanto, a partir dos resultados deste estudo, pode-se considerar que a radiografia modificada para a articu- lação temporomandibular e a radiografia panorâmica dos maxilares são bons métodos de imagem para visualizar as alterações morfológicas da articulação temporomandibular, incluindo o processo estilóide. A grande limitação deste exame é a impossibilidade de mensuração da extensão do processo, entretanto, este fator não é determinante na elaboração do diagnóstico da síndrome ${ }^{(17)}$ (Figura 2).

No universo amostral desta pesquisa, verificou-se que $15,7 \%$ dos pacientes são do sexo masculino e $84,3 \%$ são do sexo feminino, estando a maior concentração $(65,2 \%)$ dos pacientes entre 21 e 50 anos de idade. Ambos os resultados ratificam os relatos de Okeson ${ }^{(\mathbf{1 4})}$ e Saad e Barros ${ }^{(\mathbf{1})}$ sobre as desordens temporomandibulares.

Neste trabalho, 5,53\% dos pacientes com desordem temporomandibular apresentaram pelo menos um dos lados da articulação temporomandibular com alteração morfológica do processo estilóide ${ }^{(\mathbf{1 8})}$. Entretanto, Zaki et al. ${ }^{(\mathbf{1 9 )}}$ encontraram, em seu estudo, também em pacientes com desordem temporomandibular, incidência bem mais significativa (27\%). Para Eagle ${ }^{(\mathbf{1 7})}$, em 


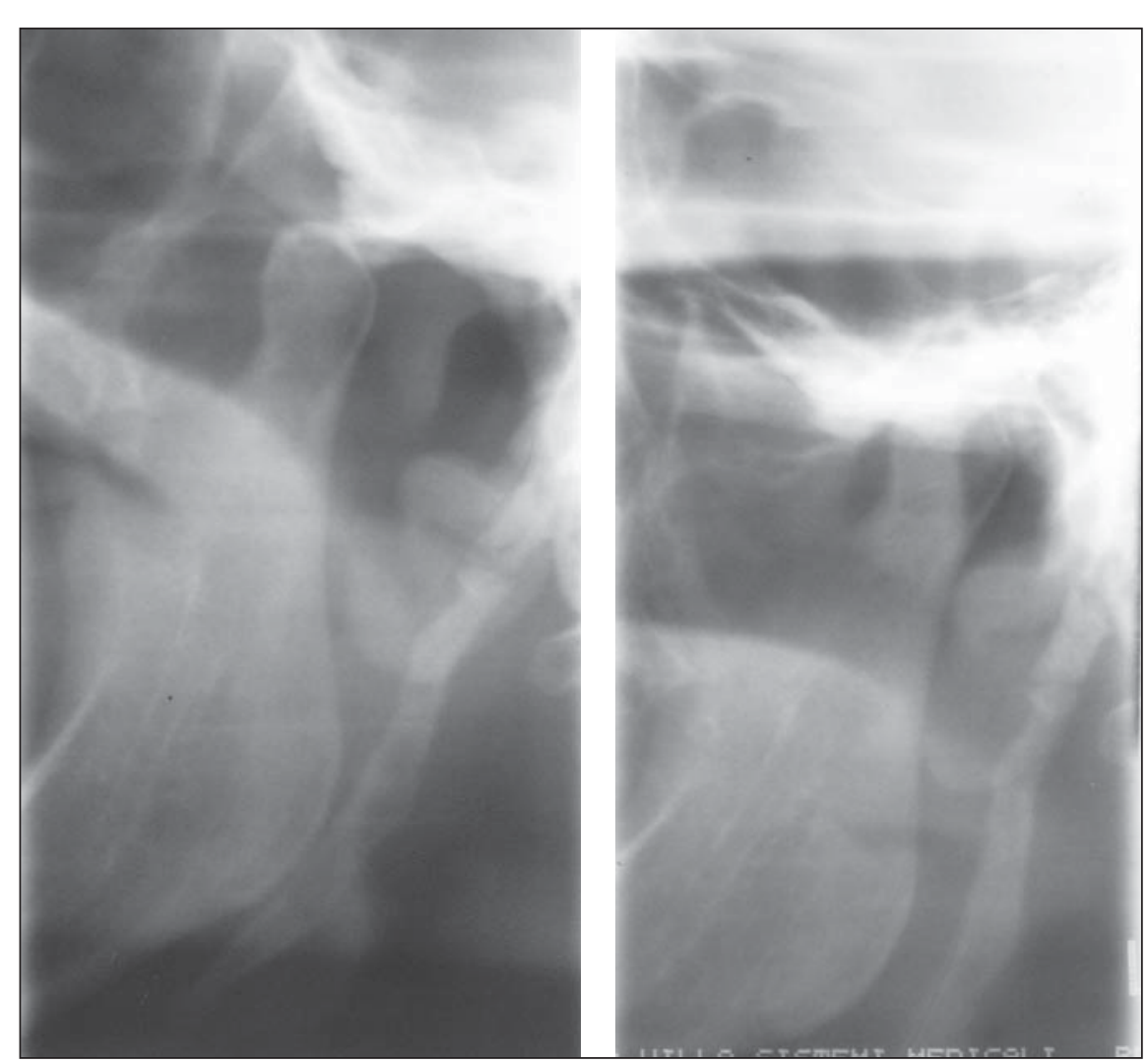

Figura 2. Imagem radiográfica da articulação temporomandibular (lado esquerdo) evidenciando a alteração morfológica do processo estilóide.

4\% dos pacientes com alteração morfológica do processo estilóide somente $4 \%$ apresentam sintomas compatíveis com a síndrome de Eagle.

Em números absolutos, houve maior incidência de alteração morfológica do processo estilóide em mulheres (74) em relação aos homens (9). Entretanto, de acordo com o teste $\mathrm{V}$ de Cramer, constatou-se que a prevalência é semelhante entre os sexos. Este resultado difere dos achados de Gozil et al. ${ }^{(20)}$, Eagle ${ }^{(\mathbf{1 0})} \mathrm{e}$ Aral et al. ${ }^{(21)}$, que afirmaram que a maior incidência é nos homens, como também difere de Hernandez et al. ${ }^{(22)}$, que encontraram maior prevalência nas mulheres.

Neste estudo, 32,5\% dos pacientes apresentaram alterações morfológicas do processo estilóide e concentravam-se entre 41 e 50 anos de idade para ambos os sexos. Nas demais faixas etárias (21-30 anos, 31$40,51-60$ anos e 61-82 anos) houve uma média de $15 \%$ cada uma. Este resultado está em desacordo com os estudos de Zaki et al..$^{(\mathbf{1 9})}$ e Aral et al. ${ }^{(21)}$ (31 a 40 anos), Basekim et al. ${ }^{(\boldsymbol{( 6 )}}$ (34 anos) e Gozil et al. ${ }^{(20)}$
(31 a 50 anos). Na faixa etária de 11 a 20 anos, neste estudo, constatou-se somente a presença de cinco pacientes, ou seja, $6 \%$ da amostra. Ainda neste estudo, num total de 1.500 pacientes, não se encontrou nenhum paciente com menos de dez anos de idade com alteração morfológica do processo estilóide; todavia, na literatura consultada ${ }^{(8)}$ encontrou-se um relato de caso de uma paciente com dez anos de idade.

Com esta grande variação na presença de alterações morfológicas do processo estilóide por faixas etárias, deve-se considerar, apoiado em diversos estudos ${ }^{(6,18,21,23)}$, inclusive no ora apresentado, que o alongamento do processo ou mineralização do complexo estilohióideo desenvolve-se de maneira particular em cada indivíduo, independentemente do sexo e da idade ${ }^{(23)}$.

A maioria dos estudos encontrados na literatura sobre alterações morfológicas do processo estilóide é relato de caso clíni$\mathrm{co}^{(8,10-12,15,21,22,24)}$ e não avalia a morfologia do processo estilóide, e sim, a parte sintomatológica e a presença do alongamento do processo.
Baseado nos estudos de Langlais et $a l^{(7)}$, no grupo amostral composto por 83 pacientes, realizou-se a avaliação do tipo morfológico de cada processo estilóide. $\mathrm{O}$ padrão alongado foi o mais freqüente (113), os demais tipos morfológicos não mostraram diferenças significantes (normal, pseudo-articulado e segmentado), confirmando os achados de diversos autores ${ }^{(6,20,23)}$. Em concordância com Aral $e t$ $a l .{ }^{(21)}$, neste estudo não houve predominância de um dos lados na alteração morfológica do processo estilóide.

Vale ressaltar a importância do diagnóstico nas disfunções craniomandibulares, através de exames clínicos e por imagens $^{(6,9,13,14,16)}$, objetivando estabelecer o diagnóstico final, uma vez que o tratamento para as desordens temporomandibulares é primeiramente sintomático, com terapias conservadoras e reversíveis ${ }^{\left({ }^{(9)}\right)}$, e o tratamento para a síndrome de Eagle envolve a excisão cirúrgica ${ }^{(5,20,22,24,25)}$, com acesso intra ou extra-oral ${ }^{(24)}$ para remoção do processo estilóide alongado ou do ligamento estilohióideo calcificado. Entretanto, Camarda et al. ${ }^{(25)}$ afirmaram que muitos pacientes podem tolerar os sintomas do processo estilóide alongado e não se submeterem à cirurgia.

Em concordância com diversos autores $^{(1,5,24)}$, acredita-se que nas dores orofaciais são necessárias a intervenção e a cooperação interdisciplinar de radiologistas, otorrinolaringologistas, cirurgiões-dentistas, neurologistas, ortopedistas, oftalmologistas, fonoaudiólogos, fisioterapeutas e psicólogos ${ }^{(16)}$, cada profissional atuando dentro da sua especialidade para definição do diagnóstico e conduta terapêutica adequadas aos casos clínicos.

\section{CONCLUSÕES}

A prevalência de alteração morfológica do processo estilóide, vista radiograficamente, nos pacientes com desordens temporomandibulares foi de $5,53 \%$, sendo $10,8 \%$ (9 pacientes) do sexo masculino e $89,2 \%$ (74 pacientes) do sexo feminino. A maior frequiência foi encontrada na faixa etária de 41 a 50 anos $(32,5 \%)$.

Os tipos morfológicos, vistos radiograficamente, do processo estilóide do osso temporal foram: 113 alongados, 21 pseudo- 
articulados e 19 segmentados, totalizando 153 processos estilóides dos 83 pacientes, sendo 13 considerados normais. Verificouse, ainda, que há correlação entre o padrão direito com o esquerdo, ou seja, há simetria da alteração morfológica.

\section{REFERÊNCIAS}

1. Saad PA, Barros JJ. In: Barros JJ, Rode SM. Tratamento das disfunções craniomandibulares ATM. 1ª ed. São Paulo: Livraria Editora Santos, 1995;341-345.

2. Ash MM, Ramfjord SP, Schimideserder J. Oclusão. 1a ed. São Paulo: Livraria Editora Santos, $1998 ; 55$.

3. Rodrigues JRC, Chilvarquer F. Avaliação clínica e radiográfica de pacientes portadores de apófise estilóide alongada e calcificada e com disfunção dolorosa da ATM. Anais SBPqO 1991;7:57.

4. Rocha APF, Nardelli MR, Rodrigues MF. Epidemiologia das desordens temporomandibulares: estudo da prevalência da sintomatologia e sua inter-relação com a idade e o sexo do pacientes. Rev Serviço-ATM-FO/UFJF 2002;2:1:5-9.

5. Noronha MJR, Gandelmann I, Araújo Jr GP, Shunemann WG. Alongamento do processo estilóide. Síndrome de Eagle. Rev Bras Otorrinolaringol 1987;53:60-63.

6. Basekim CC, Mutlu H, Gungor A, et al. Evaluation of styloid process by three-dimensional com- puted tomography. Eur Radiol 2005;15:134-139.

7. Langlais RP, Langland OE, Nortjé CJ. Diagnostic imaging of the jaws. 1st ed. Baltimore: Williams \& Wilkins, 1995;620-622.

8. Galán IR, Galán CR, Diez FA. Sindrome estilohióideo en la infancia. An Esp Pediatr 1999;50: 507-508.

9. Lee HA. Three-dimensional computed tomography imaging of Eagle's syndrome. Am J Otolaryngol 2004;25:109.

10. Eagle WW. Elongated styloid process. Report of two cases. Arch Otolaryngol 1937;25:584-587.

11. Reis SSPM, Carvalho PL, Reis HSM. Processo estilóide alongado - relato de dois casos. J Bras Oclus ATM \& Dor Orofac 2001;1:296-300.

12. Slavin KV. Eagle syndrome: entrapment of the glossopharyngeal nerve? Case report and review of the literature. J Neurosurg 2002;97:216-218.

13. Arellano JCV. Metodologia no diagnóstico da disfunção da articulação temporomandibular. J Bras Oclus ATM \& Dor Orofac 2002;2:78-86.

14. Okeson JP. Fundamentos de oclusão e desordens temporomandibulares. $4^{\mathrm{a}}$ ed. São Paulo: Artes Médicas, 2000;119-121.

15. Heitz C, Rocha JRM, Souza PHC, Costa NP. Mineralização atípica do complexo estilóide: relato de caso. Rev Bras Prótese e Implante 1999;6: 31-37.

16. Sá ACD, Zardo M, Paes Jr AJO, et al. Alongamento do processo estilóide (síndrome de Eagle): relato de dois casos. Radiol Brasil 2004;37:385387.
17. Eagle WW. Elongated styloid process; symptoms and treatment. Arch Otolaryngol 1958;67:172176.

18. Goldstein GR, Scopp IW. Radiographic interpretation of calcified stylomandibular and stylohyoid ligaments. J Prosthet Dent 1973;30:330-334.

19. Zaki HS, Greco CM, Rudy TE, Kubinski JA. Elongated styloid process in a temporomandibular disorder sample: prevalence and treatment outcome. J Prosthet Dent 1996;75;399-405.

20. Gozil R, Yener N, Çalguner E, Araç M, Tunç E, Bahcelioglu M. Morphological characteristics of styloid process evaluated by computed axial tomography. Ann Anat 2001;183:527-535.

21. Aral IL, Karaca I, Gungor N. Eagle's syndrome masquerading as pain of dental origin. Case report. Aust Dent J 1997;42:18-19.

22. Hernandez C, Rodriguez MM, Sano R, Vargas S, Monasterio MV. Síndrome de Eagle: a propósito de un caso. Acta Otorrinolaringológica 2000;12: 57-62.

23. Diamond LH, Cottrell DA, Hunter MJ, Papageorge M. Eagle's syndrome: a report of 4 patients treated using a modified extraoral approach. J Oral Maxillofac Surg 2001;59:1420-1426.

24. Trevisan DRS. Análise dos aspectos do complexo estilohióideo na radiografia panorâmica. J Bras Oclus ATM \& Dor Orofac 2002:2:105-115.

25. Camarda AJ, Deschamps C, Forest D. I. Stylohyoid chain ossification: a discussion of etiology. Oral Surg Oral Med Oral Pathol 1989;67:508514 